\title{
Las cooperativas y su influencia en la capacidad de innovación de las destinaciones turísticas ${ }^{1}$
}

\author{
Cooperatives and Their Influence on the Innovation \\ Capacity of Tourist Destinations
}

Lluís Prats Planagumä

\section{Resumen}

Las cooperativas turísticas aparecieron a principios de los sesenta para hacer frente a un nuevo fenómeno de masas que ejercía una presión excesiva sobre los agentes turísticos, los cuales tenían que soportarla individualmente. Su constante evolución en paralelo con la de las destinaciones turísticas y también con la de las necesidades de los turistas está transformando estas cooperativas y dirigiéndolas de manera forzosa hacia fases estratégicas más avanzadas. Son estas nuevas fases, junto con los nuevos conceptos de gestión de las destinaciones, las que obligan a poner énfasis en la mayor capacidad de innovación que son capaces de generar las cooperativas frente a las destinaciones turísticas que no tienen estas estructuras desarrolladas. En este trabajo se explican las razones básicas por las que las cooperativas pueden acceder a estas fases estratégicas avanzadas, aumentando así su capacidad de innovación. Dicho resultado es producto de los hallazgos de investigación sobre "Gestión de destinaciones" del grupo de investigación "Organizational Networks, Innovation and Tourism (oNIT)" de la Facultad de Turismo de la Universidad de Girona, 2011.

Palabras clave: agentes, capacidad de innovación, colaboración, diferenciación, turismo.

\section{Abstract}

The tourism cooperatives appeared in the early sixties to meet a new mass phenomenon that exerted excessive pressure on travel agents to be supported individually. Their constant evolution, in parallel with that of the tourist destinations and also with the needs of the tourists, is transforming these cooperatives and directing them forcibly towards more advanced strategic phases. These are the new phases, along with the new concepts of management of destinations, which require the greater emphasis on innovation capacity generated by cooperatives over tourist destinations without these developed structures. This paper explains the basic reasons that cooperatives can access these advanced strategic phases, increasing its capacity for innovation. This result is the product of research

1 Artículo de reflexión derivado de los hallazgos de investigación sobre "Gestión de destinaciones" del grupo de investigación "Organizacional Networks, Innovation and Tourism (ONIT)" de la Facultad de Turismo de la Universidad de Girona, 2011.

Doctor en Ciencias Económicas de la Universidad de Toulouse 1. Doctor en Administración y Dirección de Empresas de la Universidad de Jaume I de Castelló de la Plana. Profesor titular de la Universidad de Girona. Correo electrónico: lluis.prats@udg.edu

Cómo citar este artículo: Prats Planagumà, L. (2012). Las cooperativas y su influencia en la capacidad de innovación de las destinaciones turísticas. Revista Cooperativismo \& Desarrollo, 20(101), 132-144. 
findings on "Managing destinations" of the research group "Organizational Networks, Innovation and Tourism (ONIT)" of the Faculty of Tourism at the Universidad de Girona, 2011.

Keywords: agents, innovation capacity, collaboration, differentiation, tourism.

Descriptores alfanuméricos (JEL Codes): R580-Regional Development Planning and Policy; M140-Corporate Culture; Social Responsibility; Z130-Economic Sociology, Economic Anthropology, Social and Economic Stratification.

Recibido: 30 de septiembre del 2011 Aprobado: 15 de junio del 2012

\section{Introducción}

Las cooperativas pueden ser un fenómeno especialmente interesante en el sector turístico. En relación con la demanda, se constata que la variedad y concentración de la oferta desempeña una función primordial en el proceso de creación y consolidación de una destinación, así como en el proceso de compra por parte de los turistas. Además, la cooperación ofrece sinergias y ventajas a sus integrantes en aspectos como la promoción, la formación de un contingente humano cualificado compartido, la regulación del sector, las centrales de compras, o incluso los elementos de gestión capaces de ser externalizados, entre otros. No obstante, en este artículo centraremos la mirada en la innovación y en destacar los elementos positivos que las cooperativas ejercen y que permiten a una destinación turística aumentar su capacidad de innovación.

El escenario actual de incremento progresivo y continuo de la complejidad de los ecosistemas empresariales, junto con el incremento de las exigencias de los mercados, lleva a pensar que las empresas deberán prepararse para escenarios de futuro no muy óptimos, como los pensados para el desarrollo de la actividad turística, especialmente en aquellas destinaciones más maduras.

Por eso las empresas se verán obligadas a apostar por la innovación continua, a partir del refuerzo de determinadas redes o uniones empresariales en las que coexisten relaciones de competencia y cooperación al mismo tiempo, que estimulan el tejido empresarial hasta hacerlo altamente competitivo a nivel global, lo que genera mayores beneficios para la mayoría de sus integrantes (Kaufman y Rousseeuw, 2005). Cabe decir que las redes cooperativas, como por ejemplo los clústers y los factores de su creación, han sido un fenómeno tratado de forma amplia en la literatura académica, sobre todo con respecto a la dimensión de la oferta, fundamentalmente a partir de aproximaciones macroeconómicas (Porter, 1998, 2003). No obstante, también se puede destacar que la capacidad de innovación que generan se percibe de manera implícita en todas estas teorías, pero no se ha tratado de forma explícita hasta hace relativamente poco (Prats, Guia y Molina, 2008).

Para entender el funcionamiento cooperativo en el entorno turístico es imprescindible mencionar el papel determinante que el territorio ejerce, territorio 
más conocido turísticamente como destinación. Así pues, la primera parte del presente artículo intenta explicar el concepto de destinación turística como territorio favorecedor de la cooperación.

En toda cooperativa existe un tipo de agente que prevalece en cuanto a número por encima de los otros tipos. No obstante, la presencia de todos ellos es lo que enriquece el conjunto cooperativo. Entonces creemos necesario repasar cuáles son los agentes que intervienen en una cooperativa turística para definir la estructura ideal de una destinación.

La interacción de estos agentes es lo que conforma la red relacional que se teje de forma indisociable en cualquier cooperativa. Por este motivo daremos un leve repaso a las teorías relacionales, dando énfasis a la característica más destacada de las redes generadas en las cooperativas, que es la existencia no sólo de relaciones puramente comerciales y personales, sino del incremento con las familiares que generan vínculos más estrechos y fuertes que los anteriores.

\section{Noción de destinación turística}

Toda destinación turística se basa en un territorio concreto en el cual interactúa e interviene el conjunto de agentes turísticos que están presentes y que tienen como factores determinantes los elementos relacionales; estos, unidos a las variables del macroentorno, permiten generar conocimiento y aprendizaje colectivo, esenciales para la capacidad de innovación del sistema (Prats y Guia, 2005).

La destinación ha dejado de ser un marco en el cual sólo suceden fenómenos económicos, y ha pasado a ser un factor importante de las organizaciones. Las destinaciones se convierten en el marco de localización de los agentes económicos, pero sobre todo el marco de un actor particular y emergente: el territorio.

Entendemos el territorio como una construcción colectiva no necesariamente ligada a la proximidad geográfica ni a la aglomeración. Se puede ver el territorio como un campo de acción en el cual los conflictos, las negociaciones, los dispositivos formales e informales de cooperación, de arbitraje de conflictos o de regulación de los diferentes agentes, forman un complejo conjunto difícil de entender desde las diversas aproximaciones académicas (Courlet, 2001). Por esta razón, es necesario entender el territorio desde una óptica multidisciplinaria como la que puede ofrecer el turismo, lo cual nos trae a constatar la importancia que puede ejercer este territorio en la industria turística.

El territorio, pues, queda concebido como un espacio en el que existe una aglomeración importante de agentes, y también se entiende que no responde necesariamente a fronteras físicas ni políticas preestablecidas. Esta definición explica que la destinación turística se comprende como una construcción socioespacial caracterizada por una idea de región construida socialmente, que va más allá de los aspectos físicos o administrativos (Giddens, 1985).

Equiparando el territorio turístico con una destinación, es posible definirlo como un sistema abierto que en su conjunto determina una oferta capaz de atraer turistas (Rodríguez-Díaz y Espino, 2008). También se entiende como un 
sistema complejo y adaptable en el que tienen lugar numerosas interrelaciones en distintos ámbitos, como el ambiental, el humano, el natural y el económico. Según Gilbert (1960), las “regiones son como individuos, tienen caracteres muy distintos y estos se desarrollan y cambian constantemente". Así, las destinaciones se pueden entender como productos en constante cambio, derivados de una cierta combinación de aspectos sociales, políticos y económicos que son específicos en el espacio y tiempo (Getz, 1999). Ritchie y Crouch (2000) explican en su modelo de competitividad de destinaciones turísticas cuáles son los elementos clave para su éxito en cuanto a capacidad de ofrecer la experiencia turística que buscan sus visitantes, los cuales se pueden resumir en: los recursos y la infraestructura; las condiciones coyunturales; la imagen proyectada por la demanda; la gestión de la destinación y, finalmente, la capacidad de esta de ofrecer los productos que la demanda exige.

\section{Los agentes relevantes en la destinación cooperativa}

Los agentes turísticos son una parte esencial e indispensable de cualquier destinación, y es por esta razón que es necesario emplear una parte importante del tiempo a identificar estos agentes. $\mathrm{Si}$ nos fijamos en las diversas definiciones de destinaciones que se han dado y las juntamos con las definiciones de cooperativas, de sistemas de innovación, de clústers, de milieux innovateurs o de distritos industriales, entre otros, aplicados a la industria tradicional, veremos que siempre nos muestran tres agentes principales que creemos importantes y que detallaremos en este apartado.

Sin embargo, pensamos que en el caso turístico también entra en juego un cuarto agente principal que ayuda a definir la situación del sistema y, por consiguiente, lo completa (figura 1). Estos agentes principales tienen en todo momento el apoyo de otros dos tipos de agentes secundarios que también analizaremos.

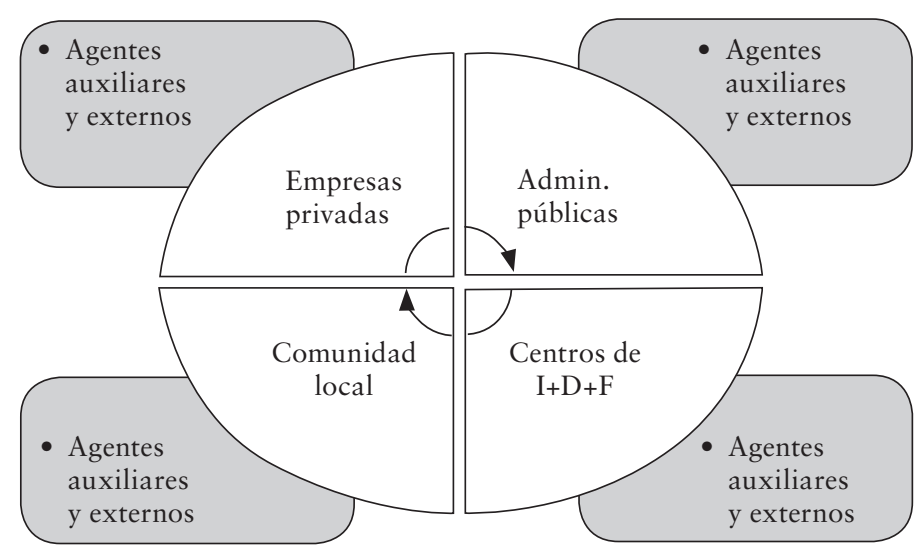

Figura 1. Agentes turísticos de una destinación cooperativa

Fuente: el autor 
El primero de estos agentes y el más habitual en la conformación de cooperativas es la empresa privada. Entendemos como empresas privadas turísticas aquellas entidades con ánimo de lucro que intervienen de forma directa en la generación de la experiencia turística. De esta manera, se incluyen las empresas turísticas básicas como las de alojamiento, restauración, entre otras, igual que aquellas que, sin ser eminentemente turísticas, basan toda su actividad en el turismo, y que sin los ingresos provenientes del turismo no serían capaces de sobrevivir, como por ejemplo las de limpieza, construcción o lavanderías, entre otras.

Como segundo agente principal nos referimos a las administraciones públicas, que a menudo tienen un papel relevante en las cooperativas turísticas, especialmente en control normativo e integrador promocional. Hablamos de administraciones públicas cuando nos referimos a aquellos organismos de carácter público que intervienen en los procesos turísticos. La transversalidad del turismo aporta una idiosincrasia diferente de la existente en otros sectores industriales. Si tomamos una institución local, como por ejemplo un ayuntamiento, y la diseccionamos, podemos observar elementos como el urbanismo, el medio ambiente, la cultura o los deportes, que adquieren por separado, pero también en su conjunto, relevancia en el campo del turismo. Esto implica que las decisiones se deberían tomar desde una visión integrada, cosa que no sucede en la mayoría de los casos.

El tercer de estos agentes principales son los centros de investigación, desarro- llo y formación $(\mathrm{I}+\mathrm{D}+\mathrm{F})$ en turismo. Nos referimos a ellos como elementos esenciales para cualquier industria, a pesar de que en el caso turístico están poco valorados y, por consiguiente, infrautilizados. Algunos ejemplos de este tipo de agentes son las universidades, los institutos de investigación, los laboratorios de empresas, las consultoras, las escuelas de formación profesional, los observatorios, etcétera. Para que estos centros formen parte del sistema cooperativo tienen que generar formación o investigación en el ámbito del turismo que pueda ser recogido y usado por los otros miembros integrantes de la cooperación.

Podemos ver que los tres agentes principales que hemos mencionado hasta ahora son los que la literatura académica sobre las cooperativas o los sistemas de innovación considera básicos. Pero nosotros, tomando como base el esquema turístico propuesto por Gunn (1997) y posteriormente adaptado por la OMT (1999), conceptualizamos la industria turística como un sistema turístico funcional (Prats, 2003).

Con esta base demostramos que la comunidad local también ejerce un papel esencial en el desarrollo de la actividad turística y, por consiguiente, son el cuarto agente principal. Podemos definir la comunidad local como el conjunto de personas que viven habitualmente en el territorio turístico. Estas personas las podemos considerar de manera individual o como partícipes de organizaciones sin ánimo de lucro, como por ejemplo ONG, plataformas ciudadanas, entidades cívicas, etcétera. 
Destacamos la importancia de la comunidad local en el turismo viendo qué movimientos comunitarios han conseguido mantener sin urbanizar determinadas zonas de interés ecológico, cultural o paisajístico, o han pedido la creación de espacios protegidos o la señalización de senderos. Estos y otros hechos pueden encaminar, frenar o impulsar el turismo, y pueden convertir la comunidad local en un potente aliado si la cooperativa funciona correctamente, o en un enemigo si no se tiene en cuenta.

También se debe tener en cuenta que las cooperativas tradicionalmente se nutren de agentes en su mayoría privados, pero que forman parte al mismo tiempo de la comunidad local, siendo en este caso más difícil aún no considerar a la comunidad local como agente principal, ya que la comunidad local ejerce una marcada influencia en la cooperativa.

Una vez descritos los elementos básicos, definimos los agentes auxiliares del turismo como todos aquellos que, a pesar de que no realicen actividades directamente relacionadas con la industria turística, le prestan algún servicio a esta. Si tomamos las teorías económicas como referente, son todas aquellas empresas receptoras del efecto multiplicador turístico, como por ejemplo comercios u oficios, entre otros. En cambio, cuando hablamos de agentes externos a la cooperativa, nos referimos a aquellos elementos que no forman parte directa de ella, aunque estos agentes pueden tener relación con uno o más miembros con los que interactúan.

Algunos autores integran otro agente principal, concretamente el sector finan- ciero (Montobbio, 1999). Nosotros pensamos que las empresas del sector financiero son muy relevantes para la innovación, y el caso turístico no es una excepción, a pesar de que no vemos el conjunto de estas empresas como un agente independiente. En nuestro supuesto, el sector financiero puede integrarse en dos de los agentes ya mencionados, según su naturaleza: empresas privadas o agente auxiliar.

Inicialmente colocaríamos una entidad financiera como agente auxiliar de la industria turística, en cuanto a que es necesaria para financiarla. En este caso estaríamos hablando de una entidad financiera que esporádicamente puede prestar servicios a un agente turístico. Sin embargo, si esta entidad financiera surge de la cooperativa, o por esta, y además se convierte en su principal fuente de trabajo, consideramos que es parte del conjunto empresarial turístico que conforma la cooperativa. Un caso ejemplar son sin duda las cajas rurales surgidas de cooperativas agrícolas.

\section{Redes relacionales}

De las definiciones anteriores es posible extraer que las cooperativas turísticas se basan mayoritariamente en una destinación concreta, y que pueden estar integradas o bien por un solo grupo de agentes, o por varios de ellos, pero en cualquiera de los dos casos la naturaleza del turismo empuja a que todos los agentes interactúen con la cooperativa, ya sea directa o indirectamente. Es en este punto en el que las redes relacionales despiertan su interés. 
El estudio de las redes relacionales se puede vincular con diversas aproximaciones teóricas dentro los campos de la economía o la sociología, entre otros (Sorensen, 2004). Así, en el terreno más estático se cree que dentro de las redes, las empresas individuales disfrutan de enlaces importantes con otras empresas, es decir que estas no son simplemente una unidad dentro de un mercado atomizado (Håkansson y Snehota, 1995), y por consiguiente deben analizarse considerando sus relaciones con otras empresas y las relaciones existentes entre terceras empresas de la red (Holmen, Pedersen y Torvatn, 2005).

Se pueden definir las redes empresariales como el conjunto de relaciones empresariales conscientes y aceptadas, ya sean formales o informales, de transmisión de recursos, materiales o inmateriales, en el ámbito de la empresa (Sorensen, 2004). Resulta, pues, útil el análisis de redes sociales que estudia relaciones específicas entre una serie definida de elementos, ya sean personas, grupos, organizaciones, países o acontecimientos (Molina, 2001).

Las relaciones existentes dentro de las redes sociales influyen las acciones económicas. Porter (1998), con el modelo de las cinco fuerzas y la posterior aproximación a los clústers, ha universalizado la necesidad de mantener los tipos de vínculos profesionales o comerciales, y otras teorías como los distritos industriales dejan clara la necesidad de contar con las relaciones personales como un valor que refuerza el conjunto de agentes, haciendo más estrecho y eficiente el vínculo entre ellos (Becattini, 2004). En nuestra opinión, estos tipos de vínculos intervienen de forma positiva en la generación de confianza entre los participantes de la red, en este caso la cooperativa, y en la asunción de mayor Responsabilidad Social Corporativa (CSR) de las redes, ya que parte de las relaciones comerciales entre los agentes están apoyadas por las personales, y también, aunque en menor medida, con los agentes externos.

Todo este conjunto de vínculos nos ponen de manifiesto el componente central de las cooperativas que relaciona los agentes con sus stakeholders, los cuales son definidos como individuos o grupos que se sienten afectados por la ejecución de los objetivos de una organización (Preble, 2005), y, consecuentemente, no son otra cosa que miembros de una red en la que varias organizaciones se relacionan.

Así, podemos establecer que, atendidas las relaciones entre una organización y sus stakeholders - los cuales a la vez son otras organizaciones que tienen otros stakeholders y forman parte de una red de agentes-, la práctica de principios de confianza y solidaridad por uno o más miembros de esta puede conducir al seguimiento del resto de miembros. Es por esta razón que seguidamente desarrollaremos el concepto de capacidad de innovación, entendiéndolo como el mayor y mejor resultado del aprovechamiento de las relaciones que tenemos tanto a nivel individual como a nivel colectivo de cooperativa. 


\section{Las cooperativas y la generación de capacidad de innovación}

El territorio por sí solo no tiene las condiciones suficientes para la coordinación y la proximidad no genera por sí sola sinergias, pero puede contribuir a su eficacia si existen otras dimensiones compartidas entre los agentes como planes o proyectos comunes, entre otros (Zimmermann, 2002).

La eficacia de las interacciones a menudo está determinada por una proximidad geográfica, puesto que permiten a los agentes beneficiarse conjuntamente de infraestructuras, ingenierías comunes, transferencia de conocimientos tácitos, etcétera, al igual que permite efectuar una serie de combinaciones productivas e interacciones más difíciles de llevar a cabo en dispersión. Aun así, en esta fase se sigue compitiendo, como podemos ver en la figura 2, y se basa en la relación comercial de competencia.

Este primer contacto acarrea que la confianza existente entre los agentes sea muy baja y frágil. En esta fase es fácil que se produzca el contacto negativo entre dos agentes de la cooperativa y la reacción que se genera es inmediata en el momento en que hay alguna actuación de aprovechamiento de uno de ellos que hace peligrar la posición en el mercado del otro. Además, los objetivos marcados por esta pequeña interactuación sólo se plantean a partir de la situación financiera de los agentes y de sus resultados, de forma que los únicos beneficiarios de las actuaciones derivadas de este tipo de relaciones son los mismos agentes que los desarrollan.
Sin embargo, toda cooperativa de ámbito local tiene que mantener agentes dispersos o externos para obtener contactos que le permitan beneficiarse de tecnologías o sistemas de gestión, entre otras, radicados fuera del ámbito local y casi imposibles de conseguir sin esta dispersión. Rallet y Torre (2001) nos hablan de los agentes situados, que son los que están dentro del sistema pero a la vez lejos de este, trayendo la noción de proximidad fuera del espacio físico. En el caso turístico podríamos estar hablando de cooperativas que integran agencias de viaje emisoras de los países que envían turistas a la destinación.

También cabe destacar que en esta primera fase de aglomeración los agentes externos pueden favorecer o limitar el cooperativismo. En el caso español, por ejemplo, la presión ejercida por los tour operadores extranjeros que pedían constantes reducciones de precio en destinaciones de costa, generaron en destinaciones como la Costa Brava la necesidad de crear cooperativas turísticas para luchar contra este efecto. Sin embargo, en otras destinaciones, como por ejemplo en Tenerife, se produjo el efecto contrario, generando una lucha entre hoteleros locales. El resultado actual muestra cómo en la Costa Brava se impidió colectivamente la entrada de grandes cadenas hoteleras manteniendo la esencia de cooperativa con pequeñas empresas locales, mientras que en Tenerife predominan los resorts de grandes cadenas hoteleras. 


\section{$1^{\text {a }}$ Fase estrategia de concentración}

Aglomeración de agentes

\section{Competición}

$2^{a}$ Fase estrategia de liderazgo en costes

Sinergias y economía de escala

Cooperación

\section{$3^{\text {a }}$ Fase estrategia de diferenciación}

Innovaciones colectivas

\section{Colaboración}

Figura 2. Fases estratégicas de competitividad de las destinaciones turísticas

Fuente: el autor

El hecho de pertenecer a una organización, como puede ser una cooperativa, se traduce en la existencia de interacciones entre sus miembros, habitualmente transformadas en rutinas de la cooperativa, permitiendo que, por consiguiente, una cooperativa se sitúe de forma directa en la fase estratégica 2 (ver figura 2). Es lo que Rallet y Torre (2001) denominan como la lógica de pertenencia. La interacción es lo que aproxima a los miembros de esta organización. Entendemos también que esta interacción será más fácil a priori si existe una base de conocimiento común, llamada lógica de similitud.

La segunda fase hace referencia a las estrategias competitivas de reducción de costes. Esta es la fase más habitual en la que se encuentran las cooperativas en nuestro entorno, puesto que es la parte en la que se establecen sinergias claras de reducción de costes. En las cooperativas turísticas las principales sinergias provienen de promoción y publicidad conjunta como destinación, en la gestión de compras o en la gestión administrativa, entre otras.

La entrada a esta fase requiere haber pasado un tiempo prudencial de contacto entre los agentes, de forma que a corto o mediano plazo sea posible que estos puedan empezar a cooperar en proyectos conjuntos, pero con un nivel de confianza todavía medio-bajo y, por tanto, el nivel de compromiso tampoco será muy elevado. Es necesario tener presente que en cualquier momento se puede producir la disolución de esta relación cooperativa si no se cumplen los objetivos para los cuales fue pensada. Los impulsores que contribuyen al establecimiento de una relación de cooperación entre agentes suelen ser organizaciones fuertes que tienen un cierto poder emprendedor y consiguen así arrastrar otros agentes de la destinación más débiles en este sentido. Los objetivos se establecen a mediano plazo y los beneficiarios son estrictamente las organizaciones participantes. 
Como la mayoría de cooperativas, las turísticas también nacen para aprovechar al unísono un conjunto de recursos que no pueden ser aprovechados individualmente por sus miembros. Sinergias y economías de escala se acompañan de un mayor poder de negociación colectivo con los proveedores y clientes. En el caso de la Costa Brava, por ejemplo, se tradujo en la creación de una cooperativa hostelera, que daba servicio a hoteles $y$ restaurantes en sus inicios, y que fue derivando, gracias al liderazgo en costes, hacia una central de compras conjunta, una central de reservas común, o a la integración de la promoción turística de la destinación.

La interacción de estos agentes genera un conjunto de elementos que son determinantes para poder definir el éxito o el fracaso de la cooperación en todos los ámbitos. Estas relaciones pueden ser de varios tipos, y las podemos resumir en dos factores clave. Dependiendo de la estructura de relaciones que se adopte en una cooperativa, variará su grado de éxito. En este factor el elemento clave es el grado de conectividad que se logre, entendiendo que cuanto más alta sea la conectividad entre los agentes de la cooperativa, mayor será el éxito.

El segundo factor hace referencia a la calidad de estas relaciones, es decir, por ejemplo, qué frecuencia tienen, y sobre todo si el contacto entre los miembros de la cooperativa genera información de provecho capaz de generar nuevo conocimiento común a partir de este intercambio de información.
Es evidente pues que crear estructuras de manera forzada puede no dar resultados positivos, por el simple hecho de que estamos forzando una estructura de relaciones que no se basa en la confianza mutua necesaria, y así la calidad de la información circulante será pobre o inexistente.

Si por el contrario, entre los miembros de la cooperativa existe una importante base de confianza, se inicia la tercera y última fase estratégica (figura 2) que es la de diferenciación. En esta los agentes cooperan y colaboran para diferenciarse como colectivo de los competidores externos a la cooperativa.

La relación de colaboración sólo es posible a largo plazo; cuando existe una elevada confianza entre los agentes, el compromiso es medio-alto y muchas veces formalizado. Los objetivos se plantean a largo plazo y suponen apostar por oportunidades esperando la obtención de resultados conjuntos. En este caso, la decisión la toman de manera compartida todos los miembros integrantes de la red de relaciones a partir del consenso. Los beneficiarios de las actuaciones colaborativas afectan positivamente a todos los actores que han participado, aunque sea en diferente medida, pero también los beneficios se pueden expandir de manera más amplia a la destinación o a la región donde están situados, aunque algunos de los beneficiarios no sean miembros de la cooperativa.

El gran salto entre la estrategia de la fase 2 y de la fase 3 es que la reducción de costes, ya sean individuales o colectivos, 
siempre tiene un límite. Este límite nos lo indica el propio producto, es decir que si reducimos los costes por debajo de este límite la calidad del producto se ve afectada, y por consiguiente la satisfacción del cliente. En el otro extremo, podemos afirmar que el hecho de diferenciarse respecto a los demás no tiene límite. Cuando se implementa un cambio o una innovación que nos hace diferentes, es evidente que los otros pueden copiarnos, o al menos intentarlo, pero aún si lo consiguen, nada impide al conjunto generar e implementar un nuevo cambio que le genere de nuevo diferenciación.

Así pues, las tres fases representan una evolución de las relaciones establecidas inicialmente, de forma que, a medida que avanza el tiempo y estas progresan, se hacen más complejas e implican un mayor grado de interacción entre los participantes. No obstante, lo más importante es que, al llegar a la tercera fase, se obtiene una capacidad de innovación elevada, gracias al cambio y a la diferenciación constante.

\section{Consideraciones finales}

Resumiendo, las estrategias competitivas de las cooperativas turísticas se basan en la aglomeración natural de agentes que el turismo genera en las denominadas destinaciones turísticas. Las necesidades de reducción de costes y de ajuste del precio en un entorno competitivo cada vez más global tienden a la generación de cooperativas de aprovechamiento de las ventajas derivadas de la colectividad.

Finalmente, y de mayor importancia, si la cooperativa es capaz de generar un elevado grado de confianza y compromiso entre sus integrantes, es posible avanzar hacia el aprendizaje colectivo y hacia la generación de nuevo conocimiento, también de modo colectivo, que deriva en el aumento de la capacidad de innovación de toda la destinación, y no sólo de los integrantes de la cooperativa. Pero si entendemos que el turista habitualmente decide sus vacaciones en función de una motivación y de una destinación concreta, nos interesa traspasar los límites de la cooperativa para que el conjunto de sus miembros puedan resultar beneficiados de la diferenciación que permite la capacidad de innovación generada.

No obstante, poder pasar de la fase estratégica de costes a la de diferenciación se debe a tres elementos: primero, a que la estructura de agentes participantes de la cooperativa necesariamente sea más amplia e integradora, es decir, teniendo en cuenta a todos los agentes, incluida la comunidad local; en segundo lugar, la confianza que debe existir entre los agentes para llevar a cabo proyectos colaborativos es mayor a la de otras fases, y esto hace que la calidad de las relaciones y de la información que circula en la red sean muy buenas, aunque esto a su vez implique que no quepan en este momento actuaciones oportunistas ni de beneficio puramente individual. Por último, como tercer elemento, esta capacidad de innovación es la que permite generar innovaciones colectivas que nos acercarán a lograr una ventaja competitiva respecto a otras destinaciones, y al mismo tiempo nos debe proporcionar riquezas colectivas que nos conduzcan hacia la sostenibilidad 
de la propia cooperativa y de la destinación para continuar evolucionando, según nuevas diferenciaciones.

\section{Referencias}

Becattini, G. (octubre, 2004). Vicissitudini e potenzialità di un concetto: il distretto industriale (Ponencia). En Becattini, G. y Sforzi, F. (Eds.). 25 Anys d'estudis sobre el districte industrial marshallià: un balanç critic. Barcelona: Universidad Internacional Menéndez y Pelayo.

Courlet, C. (2001). Terrotoires et régions les grands oubliés du développement économique. París: L'Harmattan.

Giddens, A. (1985). Time, Space and Regionalisation. Social Relations and Spatial Structures. Nueva York: St. Martin's Press. Gregory D.; Urry J. (Eds.).

Gilbert, E. W. (1960). The Idea of the Region. Geography, 45, 157-175.

Getz, D. (1999). Resort-centred Tours and Development of the Rural Hinterland: the Case of Cairns and the Atherton Tablelands. Journal of Tourism Studies, 10(2), 23-34.

Gunn, C. A. (1997). Vacationscape: Developing tourist areas. Washington D. C.: Taylor and Francis.

Håkansson, H. y Snehota, I. J. (Eds.) (1995). Developing relationships in business networks. Londres: Routledge.

Holmen, E. Pedersen, A. C. y Torvatn, T. (2005). Building relationships for technological innovation. Journal of business research, 58(9), 1240-1250.

Kaufman, L. y Rousseeuw, P. J. (2005). Finding groups in data. An introduction to cluster analysis. Nueva Jersey: John Wiley \& Sons.

Molina, J. L. (2001). El análisis de redes sociales: una introducción. Barcelona: Ediciones Bellaterra.

Montobbio, F. (1999). Istituzioni e Performance Innovativa delle Imprese: l'Approccio
Sistemico. Economia e Politica Industriale, 100, 211-257.

омт (1999). Desarrollo turístico sostenible: Guía para administradores locales. Madrid: омт.

Porter, M. E. (1998). Clusters and competition: new agendas for companies, governments and institutions. En On Competition (pp. 213-304). Boston: Harvard Business School Press.

Porter, M. E. (2003). The Economic Performance of Regions. Regional Studies, 37(6), 545-546.

Prats, L. (2003). Competitivitat i turisme: el paper rellevant del territorio. En Cicle de conferències sobre competitivitat i territori. Girona: Universidad de Girona.

Prats, L. y Guia, J. (2005). The destination as a local system of innovation. En Swarbrooke, J. (Ed). Networking and Partnership in Destinations and Development Management (pp. 91-118). Arnhem (Holanda): ATLAS.

Prats, L. Guia, J. y Molina, F. X. (2008). How tourism destinations evolve: The notion of tourism local innovation systems. Tourism \& Hospitality Research, 8(3), 178-191.

Preble, J. (2005). Towards a Comprehensive Model of Stakeholder Management. Journal of Business and Society Review Research, 110(4), 407-431.

Rallet, A. y Torre, A. (2001). Proximité géographique ou proximité organisationnelle? Une analyse spatiale des coopérations technologiques des Réseaux locallisés d'innovation. Economie Appliquée, 54(1), 147-171.

Ritchie, J. R. B. y Crouch, G. (2000). The competitive destination: A sustainability perspective. Tourism Management, 21, 1-7.

Rodríguez-Díaz, M. y Espino, T. F. (2008). A Model of Strategic Evaluation of a Tourism Destination Based on Internal and Relational Capabilities. Journal of Travel Research, 46, 368-380. 
Sorensen, F. (2004). Tourism experience innovation networks [Tesis doctoral]. Roskilde, Dinamarca: Universidad de Roskilde.
Zimmermann, J. B. (2002). Des "clusters" aux "small-worlds" une approche en termes de proximités. Géographie, Économie, Société (4), 3-17. 